\title{
Acute Bioprosthetic Mitral Valve Thrombosis: An Unfortunate Collision of Prothrombotic Risk Factors
}

\author{
Nicole M. Quattrocchi, BA, Jonathan M. Hemli, MD, S. Jacob Scheinerman, MD, Nirav C. Patel MD \\ Department of Cardiovascular and Thoracic Surgery, Lenox Hill Hospital/Northwell Health, New York, New York, USA
}

\section{ABSTRACT}

Subclinical and clinical thrombosis of bioprosthetic cardiac valves is more common than has been previously recognized. We present a unique case of acute thrombosis of a bioprosthetic mitral valve in a 40-year-old female patient undergoing hormonal stimulation as part of in vitro fertilization therapy, who also had concomitant protein $\mathrm{C}$ deficiency that was undiagnosed at the time. To the best of our knowledge, this is the first reported case of acute bioprosthetic valve thrombosis in this complex thrombophilic milieu, and suggests the need for increased screening for prothrombotic risk factors in female patients with bioprosthetic valves before they commence gonadotropin stimulation therapy.

\section{INTRODUCTION}

Bioprosthetic valve thrombosis, once thought to be a relatively rare clinical entity, is now becoming more commonly recognized, particularly in the current era of transcatheter valve and valve-in-valve therapy [Sachdev 2018]. Patients with subclinical bioprosthetic valve thrombosis may be essentially asymptomatic, with the diagnosis only being made incidentally during routine echocardiography; some of these individuals may be able to be adequately managed by anticoagulation therapy alone [Nishimura 2017]. By contrast, acute valve thrombosis can present with potentially devastating clinical consequences, requiring emergent intervention.

The hypercoagulability associated with gonadotropin therapy during in vitro fertilization (IVF) therapy is well described [Sennström 2017]. We discuss a case of acute bioprosthetic mitral valve thrombosis in the setting of a heretofore unreported combination of IVF and protein $\mathrm{C}$ deficiency.

\section{CASE REPORT}

A 40-year-old female with a background of rheumatic heart disease and prior mitral valve repair underwent subsequent

Received Fanuary 14, 2019; accepted February 8, 2019.

Correspondence: Nirav C. Pate, MD, Department of Cardiovascular and Thoracic Surgery, Lenox Hill Hospital, 130 East 77th Street, 4th Fl, New York, NY 10075; +1 (212) 434 3000; fax: +1 (212) 4344559 (e-mail: nipatel@northwell.edu). mitral valve replacement (MVR) five years thereafter with a stented porcine bioprosthesis. Regular clinical follow-up was rigorously maintained, and the patient remained asymptomatic, with a well-functioning bioprosthetic valve repeatedly demonstrated on routine serial echocardiograms.

Two years after her mitral valve replacement, the patient expressed a desire to undergo in vitro fertilization therapy. She had previously undergone numerous cycles of IVF treatment after her initial mitral valve repair procedure, without cardiac complication, although she failed to conceive at that time. Dobutamine stress echocardiography was undertaken in an attempt to unmask any subclinical valvular or left ventricular dysfunction that could become apparent in the setting of the higher metabolic demand incurred by pregnancy; the test failed to demonstrate any significant abnormalities, and the patient began receiving injections of leuprolide acetate.

A few days after commencing gonadotropin therapy, the patient presented to our institution after a 48-hour history of worsening dyspnea. Upon admission, she was found to be in frank pulmonary edema, with clinical and metabolic evidence of a low cardiac output syndrome. After emergency endotracheal intubation and institution of inotrope and pressor support, transesophageal echocardiography demonstrated multiple large masses on her bioprosthetic mitral valve leaflets, with severely restricted leaflet motion and very little flow through the valve itself (Figure 1; Video 1 https://vimeo. com/346872643). Due to progressive clinical deterioration,

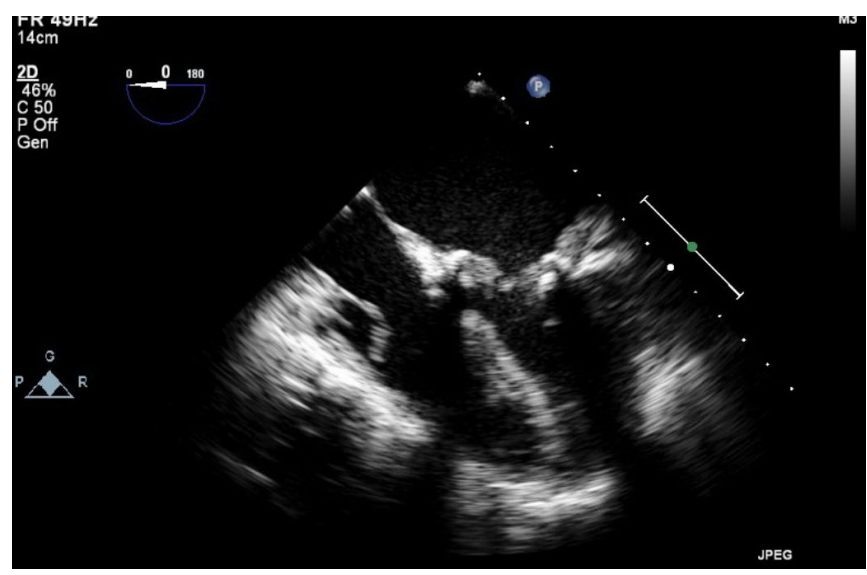

Figure 1. Still image of transesophageal echocardiogram demonstrating multiple thick masses on the bioprosthetic mitral valve causing a functional severe mitral stenosis. 


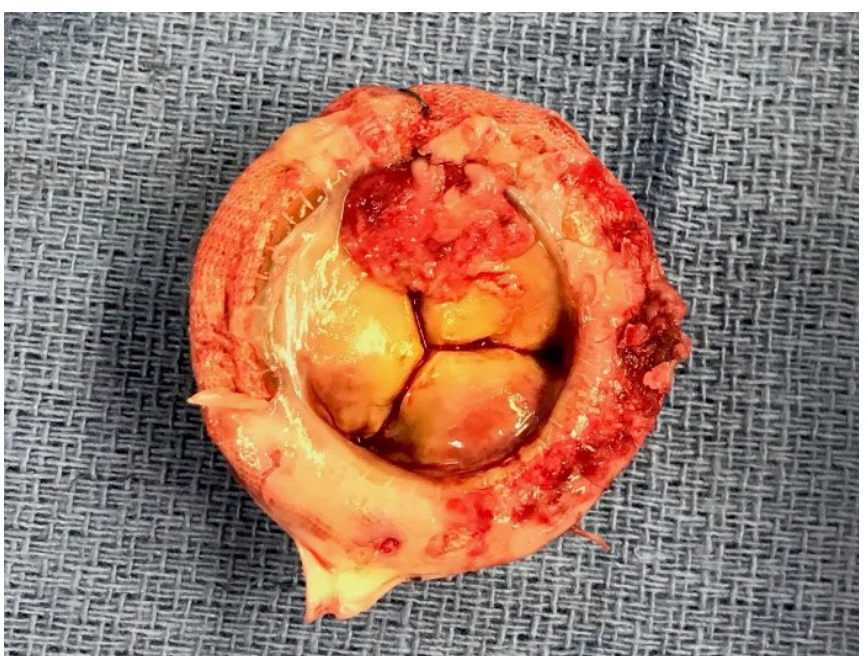

Figure 2. Explanted bioprosthetic mitral valve: a large thrombotic mass is evident crossing the atrial aspect of two of the valve leaflets.

veno-arterial extracorporeal membrane oxygenation (ECMO) was instituted via a femoral approach.

After a 24-hour period of resuscitation on ECMO, the patient underwent further reoperative mitral valve surgery. Her bioprosthetic mitral valve was found to be essentially overgrown by multiple large thrombi, particularly on the ventricular aspect of the valve leaflets (Figures 2 and 3). A new stented porcine bioprosthetic valve was implanted without complication, and the patient was successfully weaned from extracorporeal circulatory support.

Hematologic investigation during the postoperative period revealed that the patient's serum protein $\mathrm{C}$ levels were 55 percent of normal, consistent with a diagnosis of protein $\mathrm{C}$ deficiency. She was maintained on anticoagulation therapy. The patient ultimately made an uncomplicated recovery but was advised against any further attempts at IVF therapy.

\section{DISCUSSION}

The exact mechanisms underlying thrombosis of bioprosthetic valves are not, as yet, well elucidated, although the phenomenon is thought to be multi-factorial in etiology, and is likely a consequence of local hemodynamic and circulatory forces around the valve itself, coupled with various systemic patient factors, such as atrial fibrillation, obesity, diabetes mellitus, chronic renal insufficiency, smoking, anemia, and a spectrum of other low-flow states [Puri 2017]. The incidence of bioprosthetic valve thrombosis undoubtedly remains lower than that of mechanical prostheses, yet it is more common than has been previously recognized. In one of the larger series reported to date, Egbe et al identified valve thrombosis in $11.6 \%$ of 397 explanted bioprosthetic valves; more specifically, $12.7 \%$ of mitral valves demonstrated histologically proven thrombosis [Egbe 2015]. A recent study by Basra and colleagues utilized four-dimensional computed tomography to identify leaflet thrombosis in $30.9 \%$ of transcatheter aortic valve replacement (TAVR) patients and in $32.6 \%$ of patients who had undergone traditional surgical aortic valve replacement, all of whom were now presenting with either clinical or echocardiographic indicators suspicious for progressive valvular dysfunction [Basra 2018].

Bioprosthetic valves tend to be the implant of choice in women of child-bearing age that require valve replacement. Despite the increasing worldwide use of assisted reproductive technologies over the past decade [Toner 2016; Kushnir 2017], including gonadotropin stimulation, which has been consistently and reproducibly associated with an elevated risk of vascular thrombosis [Gurunath 2018], there has been only one case report to date of bioprosthetic mitral valve thrombosis in a female patient undergoing IVF therapy [Abazid 2018]. Unfortunately, not only did our patient receive hormonal stimulation, she was ultimately found to also have protein $\mathrm{C}$ deficiency, an independent marker for thromboembolic phenomena. To the best of our knowledge, this is the first description of this unlucky constellation of risk factors contributing to acute bioprosthetic valve thrombosis and circulatory collapse.

Interestingly, our patient had previously undergone IVF therapy after her index mitral valve repair surgery some years prior, without clinical or echocardiographic evidence of valvular thrombosis. Although her valve repair did ultimately fail, necessitating mitral valve replacement, there was no evidence of thrombus on the native valve leaflets or on the annuloplasty ring at the time of this operation; it was not until she had a complete bioprosthetic valve in situ that she seemed to have the requisite substrate for acute obstructive thrombosis, potentiated by underlying protein $\mathrm{C}$ deficiency and hormonal stimulation.

\section{Conclusion}

Bioprosthetic valve thrombosis is more common than has been previously recognized. Acute valve thrombosis is potentially life-threatening and warrants emergent intervention, be it

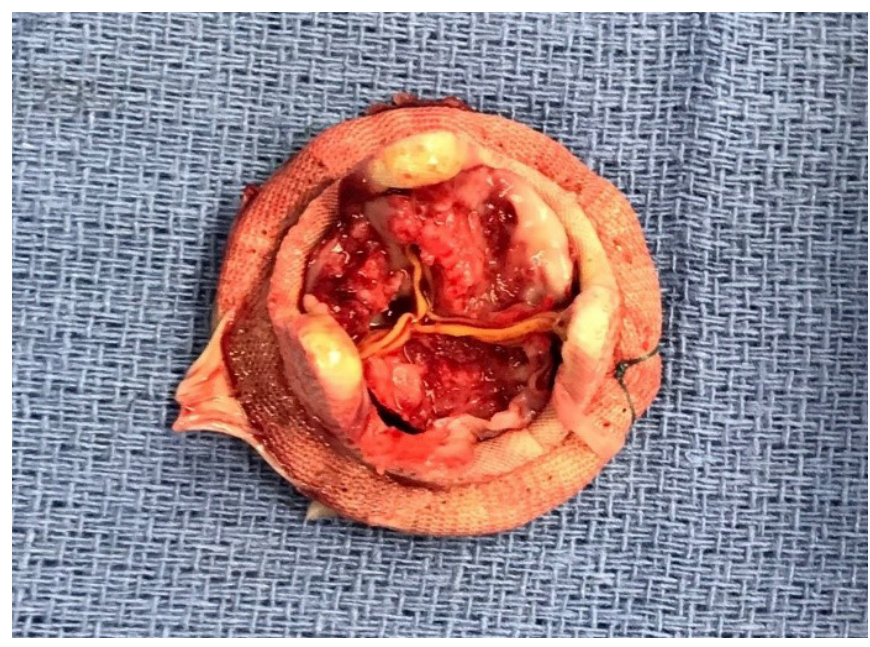

Figure 3. Explanted bioprosthetic mitral valve: thrombotic masses are evident on the ventricular aspect of all three valve leaflets, essentially occluding the valve orifice. 
surgery, lytic or transcatheter therapy. To the best of our knowledge, this is the first case reported of acute bioprosthetic mitral valve thrombosis in a patient undergoing IVF therapy who also had concomitant protein $\mathrm{C}$ deficiency. Given the inherent prothrombotic risks associated with hormonal stimulation, we suggest that all patients with bioprosthetic valves be screened for an underlying thrombophilia before commencing therapy.

\section{REFERENCES}

Abazid RM, Shoman M, Smettie OA, Elamin OA. 2018. Human menopausal gonadotropin-induced bioprosthetic valve thrombosis. Avicenna J Med 8:114-6.

Basra SS, Gopal A, Hebeler KR, et al. 2018. Clinical leaflet thrombosis in transcatheter and surgical bioprosthetic aortic valves by four-dimensional computed tomography. Ann Thorac Surg 106:1716-25.

Egbe AC, Pislaru SV, Pillikka PA, et al. 2015. Bioprosthetic valve thrombosis versus structural failure: clinical and echocardiographic predictors. J Am Coll Cardiol 66:2285-94.

Gurunath S, Vinekar S, Biliangady R. 2018. Assisted reproductive technologies in a patient with a history of venous thromboembolism: a case report and review of literature. J Hum Reprod Sci 11:193-7.

Kushnir VA, Barad DH, Albertini DF, Darmon SK, Gleicher N. 2017. Systematic review of worldwide trends in assisted reproductive technology 2004-13. Reprod Biol Endocrinol 15:6.

Nishimura RA, Otto CM, Bonow RO, et al. 2017. 2017 AHA/ACC focused update of the 2014 AHA/ACC guideline for the management of patients with valvular heart disease: a report of the American College of Cardiology/ American Heart Association Task Force on clinical practice guidelines. J Am Coll Cardiol 70:252-89.

Puri R, Auffret V, Rodes-Cabau J. 2017. Bioprosthetic valve thrombosis. J Am Coll Cardiol 69:2193-211.

Sachdev S, Bardia N, Nguyen L, Omar B. 2018. Bioprosthetic valve thrombosis. Cardiol Res 9:335-42.

Sennström M, Rova K, Hellgren M, et al. 2017. Thromboembolism and in vitro fertilization - a systematic review. Acta Obstet Gynecol Scand 96:1045-52.

Toner JP, Coddington CC, Doody K, et al. 2016. Society for Assisted Reproductive Technology and assisted reproductive technology in the United States: a 2016 update. Fertil Steril 106:541-6. 